\title{
Solution of the Boltzmann equation for primary light ions and the transport of their fragments
}

\author{
J. Kempe* and A. Brahme \\ Division of Medical Radiation Physics, Department of Oncology-Pathology, \\ Karolinska Institutet and Stockholm University, Stockholm, Sweden \\ (Received 16 February 2009; revised manuscript received 11 March 2010; published 18 October 2010)
}

\begin{abstract}
The Boltzmann equation for the transport of pencil beams of light ions in semi-infinite uniform media has been calculated. The equation is solved for the practically important generalized 3D case of Gaussian incident primary light ion beams of arbitrary mean square radius, mean square angular spread, and covariance. The transport of the associated fragments in three dimensions is derived based on the known transport of the primary particles, taking the mean square angular spread of their production processes, as well as their energy loss and multiple scattering, into account. The analytical pencil and broad beam depth fluence and absorbed dose distributions are accurately expressed using recently derived analytical energy and range formulas. The contributions from low and high linear energy transfer (LET) dose components were separately identified using analytical expressions. The analytical results are compared with SHIELD-HIT Monte Carlo (MC) calculations and found to be in very good agreement. The pencil beam fluence and absorbed dose distributions of the primary particles are mainly influenced by an exponential loss of the primary ions combined with an increasing lateral spread due to multiple scattering and energy loss with increasing penetration depth. The associated fluence of heavy fragments is concentrated at small radii and so is the LET and absorbed dose distribution. Their transport is also characterized by the buildup of a slowing down spectrum which is quite similar to that of the primaries but with a wider energy and angular spread at increasing penetration depths. The range of the fragments is shorter or longer depending on their nuclear mass to charge ratio relative to that of the primary ions. The absorbed dose of the heavier fragments is fairly similar to that of the primary ions and also influenced by a rapidly increasing energy loss towards the end of their ranges. The present analytical solution of the Boltzmann equation accurately accounts for the loss of primary particles as well as their energy losses and multiple scattering. At the same time these quantities for the fragments are also accurately derived as based on the generalized Gaussian solution of the primaries and compared both with Monte Carlo and experimental data. The results are useful for fast transport calculations and biologically optimized therapy planning with light ion beams.
\end{abstract}

DOI: 10.1103/PhysRevSTAB.13.104702

PACS numbers: $87.10 . \mathrm{Ca}$

\section{INTRODUCTION}

The unique physical and biological properties of light ion beams make them ideal for use in radiation therapy particularly when treating radiation resistant tumors using conventional low LET beams [1]. The therapeutic value of the lightest ions beyond protons such as helium and lithium has recently been discussed in terms of their depth dose and LET distributions with a clear separation between a low LET plateau and fragmentation region and a high LET therapeutic Bragg peak [2]. Similar physical and biological properties are also found in boron beams. To take full advantage of the clinical properties of light ion beams, the full three-dimensional spatial distribution is of vital importance in terms of fluence, absorbed dose, and radiation quality. Analytical relations for the radiation field

\footnotetext{
*Corresponding author. johanna.kempe@ki.se
}

quantities of the primary light ions and their associated fragments, such as fluence, mean energy, LET, and absorbed dose, are also fundamental for understanding the transport and accurate dosimetry of light ion beams. Accurate Monte Carlo simulations of the particle transport are still too time consuming for routine treatment planning. For biological treatment optimization with light ions, continuous analytical transport equations are therefore very useful for absorbed dose and radiation quality determination within the patient. Based on the simple Fokker-Plank approximation of the multiple scattering collision term of the Boltzmann equation, the first analytical solution for pencil beam transport was derived by Fermi in 1941 [3] and Eyges in 1948 [4] taking energy losses within the continuous slowing down approximation (CSDA) into account. This pencil beam solution has further been generalized to describe the lateral spread of arbitrary Gaussian beams of known initial mean square radial $\vec{r}^{2}(0)$, angular spread, $\bar{\theta}^{2}(0)$, and covariance $\bar{r}^{\theta}(0)$ [5,6], of clinical electron beams in a patient during radiation therapy of 
malignant tumors [5-8], as well as to describe the transport of light ions in different media [9-15].

More accurate and generally applicable analytical expressions, for the transport of the primary particles and their associated fragments, are still missing. They are needed not least to allow fast calculation of radiation quality and absorbed dose distributions in the patient during light ion radiation therapy. The transport of the projectiles in ion beams is almost solely determined by a strong decreasing fluence compensated by an almost proportional increase in energy loss towards the Bragg peak with increasing nuclear charge of the primaries. In addition, during the slowing down of the high-energy projectiles, fragments are continuously generated. These nuclear interactions will also have an increasing influence on the total absorbed distribution $[2,16]$. The present Boltzmann equation is solved for primary incident ion pencil beams and the solution is also used to derive broad beam distributions.

The present solution is based upon the idea of separating the primary particle transport into two components: (1) the longitudinal loss of primary particles and (2) their lateral spread. The new solution is further applied to the transport of the fragments generated by the primary particles, where the initial conditions of the generated fragments are related to those of the primary particles at the point in the beam where they are generated.

The lateral distribution function is based on a twofold Fokker-Planck expansion of the CSDA of the Boltzmann equation, within the small energy loss and deflection angle approximation. The analytical solutions are combined with recently developed accurate energy loss, range relations, and multiple scattering expressions for the primary ions and their generated fragments $[16,17]$. The loss of primaries as well as their associated secondaries is shown to be largely based on closely exponential particle fluence attenuation. The Monte Carlo code SHIELD-HIT $[18,19]$ as well as the modified version SHIELD-HIT $+[16,17]$ were used to calculate the fluence and absorbed dose for broad beams and narrow pencil beams. The code SHIELD-HIT + was used to calculate the double differential fluence in both energy and angle for plane monodirectional and monoenergetic incident ${ }^{7} \mathrm{Li},{ }^{11} \mathrm{C}$, and ${ }^{12} \mathrm{C}$ ion beams. The primary ions and their generated fragments were scored separately. An accurate 3D description of the primary ion transport is highly desirable, particularly for light ion beams such as $\mathrm{He}, \mathrm{Li}, \mathrm{Be}$, and $\mathrm{B}$, where the greater part of the dose and practically all the high LET dose components are delivered by the primary particles and the absorbed dose due to their fragments is generally of minor importance. The small tail beyond the Bragg peak in these light ion beams is of generally low dose and low LET character [2]. In beams having higher fragmentation cross section, such as ${ }^{12} \mathrm{C}$ and ${ }^{16} \mathrm{O}$, an accurate description of the transport of the heavier fragments is also of importance, since it helps to localize accurately the high LET regions, where the highest variation in the relative biological effectiveness (RBE) are expected. In addition, for positron emitting radioactive beams such as ${ }^{11} \mathrm{C}$ there is a high therapeutic interest since their range can be determined in vivo and, consequently, within the use of analytical expressions for fluence and LET, their dose distribution can be calculated and compared with that of the absorbed dose from the detected activity distribution.

The results of the analytical calculations as well as the Monte Carlo ones are compared for clinically relevant cases and found to be in good agreement.

\section{THEORY}

\section{A. The transport of projectiles in therapeutic pencil beams}

Assume that a point, monodirectional, light ion, beam is perpendicularly incident upon the surface of a homogeneous semi-infinite medium. We normalize the velocity vector $\boldsymbol{v}$ and define $\boldsymbol{u}=\boldsymbol{v} /|\boldsymbol{v}|, \boldsymbol{v} \neq 0$. Selecting the $z$ axis along the beam direction with the origin at the point of incidence, the time independent Boltzmann equation for the ion fluence $f(\boldsymbol{r}, \boldsymbol{u}, E)$ at the position $\boldsymbol{r}$ with the direction vector $\boldsymbol{u}$ of the velocity is given by

$$
\begin{aligned}
\boldsymbol{u} \cdot & \nabla f(\boldsymbol{r}, \boldsymbol{u}, E)+\mu(E) f(\boldsymbol{r}, \boldsymbol{u}, E) \\
= & N \int_{u=0}^{4 \pi} \int_{0}^{\infty} \sigma_{s}\left(E^{\prime}, E, \boldsymbol{u}^{\prime}, \boldsymbol{u}\right) f\left(\boldsymbol{r}, \boldsymbol{u}^{\prime}, E^{\prime}\right) d E^{\prime} d \boldsymbol{u}^{\prime} \\
& -N \int_{u=0}^{4 \pi} \int_{0}^{\infty} \sigma_{s}\left(E^{\prime}, E, \boldsymbol{u}^{\prime}, \boldsymbol{u}\right) f(\boldsymbol{r}, \boldsymbol{u}, E) d E^{\prime} d \boldsymbol{u}^{\prime} \\
& +Q(\boldsymbol{r}, \boldsymbol{u}, E),
\end{aligned}
$$

where $f(\boldsymbol{r}, \boldsymbol{u}, E) d E d \boldsymbol{u}$ is the fluence of ions at the position $\boldsymbol{r}$, with energy between $E$ and $E+d E$ and direction of motion between $\boldsymbol{u}$ and $\boldsymbol{u}+d \boldsymbol{u}$. Furthermore, the attenuation coefficient is given by $\mu(E)=\int N \sigma_{a}\left(\boldsymbol{u}, E, \boldsymbol{u}, E^{\prime}\right) d \boldsymbol{u}^{\prime} d E^{\prime}$, where $\sigma_{a}$ is the energy dependent macroscopic interaction cross section, $N$ is the number of target atoms per unit volume, $\sigma_{s}$ is the differential scattering cross section of ions in the Coulomb field of the nucleus, and finally $Q(\boldsymbol{r}, \boldsymbol{u}, E)$ is the source term. For the primary light ion transport, the following approximations of Eq. (1) have been applied. (i) Assuming small energy variation and making a Taylor expansion of $\sigma_{s}$ and $f$ around $E$, Eq. (1) yields

$$
\begin{aligned}
\boldsymbol{u} \cdot & \nabla f(\boldsymbol{r}, \boldsymbol{u}, E)+\mu(E) f(\boldsymbol{r}, \boldsymbol{u}, E) \\
= & N \int_{u=0}^{4 \pi} \sigma_{s}\left(E, \boldsymbol{u}^{\prime}, \boldsymbol{u}\right)\left[f\left(\boldsymbol{r}, \boldsymbol{u}^{\prime}, E\right)-f(\boldsymbol{r}, \boldsymbol{u}, E)\right] d \boldsymbol{u}^{\prime} \\
& +\frac{\partial}{\partial E}[S(E) f(\boldsymbol{r}, \boldsymbol{u}, E)]+\frac{\partial^{2}}{\partial E^{2}}[\Omega(E) f(\boldsymbol{r}, \boldsymbol{u}, E)] \\
& +Q(\boldsymbol{r}, \boldsymbol{u}, E),
\end{aligned}
$$

where $\sigma_{s}\left(E, \boldsymbol{u}^{\prime}, \boldsymbol{u}\right)$ is the differential cross section for elastic scattering, $S(E)$ is the ion stopping power, and $\Omega(E)$ is the 
energy loss straggling given by $\Omega(E)=\int_{0}^{\infty}\left(E^{\prime}-E\right)^{2} \times$ $\left[\sigma_{s}\left(E, E^{\prime}-E\right)\right] d E^{\prime}$. (ii) The small range straggling term $\frac{\partial^{2}}{\partial E^{2}}[\Omega(E) f(\boldsymbol{r}, \boldsymbol{u}, E)]$ is due to the small variation in energy losses and is disregarded for simplicity here. (iii) The source term $\mathcal{Q}(\boldsymbol{r}, \boldsymbol{u}, E)$ for the fragments transport is neglected when treating the primary ion penetration. The Boltzmann equation for the primary light ion transport is then reduced to

$$
\begin{aligned}
\boldsymbol{u} & \cdot \nabla f(\boldsymbol{r}, \boldsymbol{u}, E)+\mu(E) f(\boldsymbol{r}, \boldsymbol{u}, E) \\
= & N \int_{u=0}^{4 \pi} \sigma_{s}\left(E, \boldsymbol{u}^{\prime}, \boldsymbol{u}\right)\left[f\left(\boldsymbol{r}, \boldsymbol{u}^{\prime}, E\right)-f(\boldsymbol{r}, \boldsymbol{u}, E)\right] d \boldsymbol{u}^{\prime} \\
& +\frac{\partial}{\partial E}[S(E) f(\boldsymbol{r}, \boldsymbol{u}, E)] .
\end{aligned}
$$

This form of the Boltzmann equation [Eq. (3)] can be solved by a partial separation of the lateral transport by setting the total distribution function $f(\boldsymbol{r}, \boldsymbol{u}, E)$ equal to the product of the distribution function of the lateral displacement of the primary particles, $h(\boldsymbol{r}, \boldsymbol{u}, E)$, and the distribution function of the loss of primaries $g(E)$ according to

$$
f(\boldsymbol{r}, \boldsymbol{u}, E)=g(E) h(\boldsymbol{r}, \boldsymbol{u}, E) \neq 0 .
$$

The Boltzmann equation (3) will then be transformed to

$$
\begin{aligned}
\boldsymbol{u} \cdot & \nabla h(\boldsymbol{r}, \boldsymbol{u}, E) g(E)+\mu(E) h(\boldsymbol{r}, \boldsymbol{u}, E) g(E) \\
= & \left(N \int _ { u = 0 } ^ { 4 \pi } \sigma _ { s } ( E , \boldsymbol { u } ^ { \prime } , \boldsymbol { u } ) \left[h\left(\boldsymbol{r}, \boldsymbol{u}^{\prime}, E\right)\right.\right. \\
& \left.-h(\boldsymbol{r}, \boldsymbol{u}, E)] d \boldsymbol{u}^{\prime}\right) g(E)+g(E) \frac{\partial}{\partial E}[S(E) h(\boldsymbol{r}, \boldsymbol{u}, E)] \\
& +[S(E) h(\boldsymbol{r}, \boldsymbol{u}, E)] \frac{\partial}{\partial E} g(E),
\end{aligned}
$$

where the latter terms in Eq. (5) result from the stopping power expression in Eq. (3).

Assuming that $g(E) \neq 0$ and $h(\boldsymbol{r}, \boldsymbol{u}, E) \neq 0$ and dividing all terms by $g(E) h(\boldsymbol{r}, \boldsymbol{u}, E)$ and further rearranging the terms of Eq. (5), it is recognized that two terms have only a simple energy dependence and they are collected in the left member of the equation according to

$$
\begin{aligned}
& \frac{-\frac{\partial}{\partial E}[S(E) h(\boldsymbol{r}, \boldsymbol{u}, E)]+\boldsymbol{u} \cdot \nabla h(\boldsymbol{r}, \boldsymbol{u}, E)-\left\{N \int_{u=0}^{4 \pi} \sigma_{s}\left(E, \boldsymbol{u}^{\prime}, \boldsymbol{u}\right)\left[h\left(\boldsymbol{r}, \boldsymbol{u}^{\prime}, E\right)-h(\boldsymbol{r}, \boldsymbol{u}, E)\right] d \boldsymbol{u}^{\prime}\right\}}{h(\boldsymbol{r}, \boldsymbol{u}, E)} \\
& =\frac{\frac{\partial}{\partial E}[g(E)] S(E)}{g(E)}-\mu(E)=\varepsilon(E),
\end{aligned}
$$

where $\varepsilon(E)$ is a separation constant related to a possible remaining energy dependence of the two expressions in Eq. (6). The numerator of the first half of the Eq. (6) for the special case when $\varepsilon(E) \equiv 0$ is recognized as the wellknown Fermi-Eyges diffusion equation [4] under CSDA as discussed in more detail below. In order to compare the transport equation [Eq. (6)] with previous solutions, a scaling transformation is introduced [9]. This scaling transformation corresponds to a normalization of the transport equation of the particles as a function of normalized depth according to

$$
t(E)=R(E) / R\left(E_{0}\right)=\int_{0}^{E} d E^{\prime} /\left[S\left(E^{\prime}\right) R_{0}\right],
$$

where $t(E)$, is the relative residual path length, in relation to the continuous slowing range $R_{0}=R\left(E_{0}\right)$ of the primaries, for an initial energy $E_{0} . R(E)$ is the path length of the particles with energy $E$. In the first approximation, for the especially simple case where $\varepsilon(E) \equiv 0$, the equations for the two parts of the distribution function $f(\boldsymbol{r}, \boldsymbol{u}, t)$ reduce to

$$
\begin{aligned}
& -\left(\frac{1}{s R_{0}}\right) \frac{\partial}{\partial t}[\operatorname{sh}(\boldsymbol{r}, \boldsymbol{u}, t)]+\boldsymbol{u} \nabla h(\boldsymbol{r}, \boldsymbol{u}, t) \\
& =N \int_{u=0}^{4 \pi} \sigma_{s}\left(t, \boldsymbol{u}^{\prime}, \boldsymbol{u}\right)\left[h\left(\boldsymbol{r}, \boldsymbol{u}^{\prime}, t\right)-h(\boldsymbol{r}, \boldsymbol{u}, t)\right] d \boldsymbol{u}^{\prime}
\end{aligned}
$$

and

$$
\frac{\frac{\partial g(t)}{\partial t}}{g(t) R_{0}}=\mu(t)
$$

through use of the relations

$$
\partial h(\boldsymbol{r}, \boldsymbol{u}, t) / \partial t=[\partial h(\boldsymbol{r}, \boldsymbol{u}, E) / \partial E] S(E) R_{0}
$$

and

$$
\partial g(t) / \partial t=[\partial g(E) / \partial E] S(E) R_{0} .
$$

\section{Solution of the lateral transport $h(r, u, t)$}

The solution of Eq. (8) can be obtained by Fokker-Plank approximation, through a Taylor expansion. We let $\eta=$ $\cos \left(\boldsymbol{u}, \boldsymbol{e}_{z}\right)$, where $\boldsymbol{e}_{z}$ is the unit vector in the $z$ direction. Then using Legendre polynomial expansion in $\eta$ and following the technique used in [9] and under the assumption that the energy dependence is included as an explicit $z$ dependence [9], assuming the beam is incident along the positive $z$ axis and $\theta_{x}$ and $\theta_{y}$ are the deflection angles projected on the $z-x$ plane and the $z-y$ plane, respectively, Eq. (8) reduces to

$$
\frac{\partial h}{\partial z}+\theta_{x} \frac{\partial h}{\partial x}+\theta_{y} \frac{\partial h}{\partial y}=\frac{T}{4}\left\{\frac{\partial^{2} h}{\partial \theta_{x}^{2}}+\frac{\partial^{2} h}{\partial \theta_{y}^{2}}\right\}
$$


which is recognized as the Fermi-Eyges diffusion equation [4], where $h=h\left(z, x, y, \theta_{x}, \theta_{y}\right)$ and $T$ is the massscattering power for the primary light ions [16].

For each plane the approximation to the Fokker-Planck equation can be solved by a Fourier transform in each of the $\theta_{x}$ and $\theta_{y}$ planes. The solution of Eq. (12) can be generalized for each plane for an incident beam with a Gaussian distribution in both position and angle $[5,8,9,12]$ by

$$
h_{p, x}(z, x, \theta)=\frac{\exp \left(-\frac{\bar{\theta}_{x}^{2}(z) x^{2}-2 \bar{\theta}_{x}(z) x \theta_{x}+\bar{x}^{2}(z) \theta_{x}^{2}}{2\left\{\bar{\theta}_{x}^{2}(z) \bar{x}^{2}(z)-\left[\bar{x} \theta_{x}(z)\right]^{2}\right\}}\right)}{\pi\left\{2 \bar{\theta}_{x}^{2}(z) \bar{x}^{2}(z)-2\left[\overline{x \theta}_{x}(z)\right]^{2}\right\}^{1 / 2}} .
$$

By assuming a circularly symmetric beam and azimuthally invariant scattering $\bar{x}^{2}=\bar{y}^{2}, \bar{\theta}_{x}^{2}=\bar{\theta}_{y}^{2}$, and $\overline{x \theta}_{x}=\overline{y \theta}_{y}$, where $\bar{\theta}^{2}=\bar{\theta}_{x}^{2}+\bar{\theta}_{y}{ }^{2}$ and $\overline{r \theta}=\overline{x \theta}_{x}+\overline{y \theta}_{y}$, Eq. (13) and the associated equation in $y$ reduce to the circularly symmetric radial distribution:

$$
h_{p}(z, r, \theta)=\frac{\exp \left(-\frac{r^{2}}{\bar{r}_{p}^{2}(z)}\right)}{\pi \bar{r}_{p}^{2}(z)} \frac{\exp \left(-\frac{[\theta-\theta(r)]^{2}}{\bar{\theta}_{p, c}^{2}(z)}\right)}{\pi\left[\bar{\theta}_{p, c}^{2}(z)\right]},
$$

where $r=\sqrt{x^{2}+y^{2}}$ is the radial distance from the $z$ axis and $\bar{r}_{p}^{2}(z)=\bar{x}^{2}(z)+\bar{y}^{2}(z)$ is the mean square radius of the primary particle, given by

$$
\begin{aligned}
\bar{r}_{p}^{2}(z)= & \bar{r}_{p}^{2}(0)+2 \overline{r \theta}_{p}(0) z+\bar{\theta}_{p}^{2}(0) z^{2} \\
& +\int_{0}^{z}(z-u)^{2} T_{p}\left[E_{p}(u)\right] d u,
\end{aligned}
$$

where $\bar{r}_{p}^{2}(0)$ is the mean square initial radius of the primary particles in the beam. $\overline{r \theta}_{p}(0)$ is the initial primary particle's covariance, which quantifies the mean divergence of the primary particles outside the central axis and $\bar{\theta}_{p}^{2}(0)$ the primary particles initial mean angular spread. $T_{p}\left[E_{p}(u)\right]$ is the energy dependent scattering power at the position $u$ along the $z$ axis based on the primary particle energy depth dependence [17]. From Eq. (15) it is thus easy to realize that an increasing mass-scattering power caused by a decreasing energy will also result in an increasing value of the mean square radius. This effect is augmented with increasing penetration depth as the mean energy of the ion decreases. The depth-dependent covariance, $\overline{r \theta}_{p}(z)$, describes the most probable inclination of particles off axis and is given by

$$
\begin{aligned}
\overline{r \theta}_{p}(z)= & \overline{r \theta}_{p}(0)+\bar{\theta}_{p}^{2}(0) z \\
& +\int_{0}^{z}(z-u) T_{p}\left[E_{p}(z-u)\right] d u .
\end{aligned}
$$

Furthermore, $\bar{\theta}_{p}^{2}(z)$ describes the mean square angular spread of all primary particles in the beam,

$$
\bar{\theta}_{p}^{2}(z)=\bar{\theta}_{p}^{2}(0)+\int_{0}^{z} T_{p}\left[E_{p}(u)\right] d u,
$$

where the mean square angular spread is expected to increase with penetration depth as the mean energy of the ion rapidly decreases.

The mean square angular spread at any radial distance $r$ in the beam is independent of $r$ in the present approximation and $\bar{\theta}_{p, c}^{2}(z)$, the mean square angular spread at the central axis, is more important for dosimetric and other transport purposes than the mean square angular spread of the whole beam [8], and it is given by

$$
\bar{\theta}_{p, c}^{2}(z)=\bar{\theta}_{p}^{2}(z)-\frac{\overline{r \theta}_{p}(z)^{2}}{\bar{r}_{p}^{2}(z)} .
$$

The most probable angle of motion at a radial distance $r$ is given by

$$
\theta(r)=\frac{r}{s_{p, \mathrm{vir}}}
$$

where

$$
s_{p, \mathrm{vir}}=\frac{\bar{r}_{p}^{2}(z)}{\overline{r \theta} \theta_{p}(z)}
$$

is the distance to the virtual point source or the effective point of divergence of the beam. Hence, $\theta(r)$ is expected to decrease with increasing value of $s_{p \text {,vir }}$, and $\theta(r)$ tends to zero when the covariance $\overline{r \theta}_{p}(z)$ equals zero or when $\bar{r}_{p}^{2}(z) \gg \overline{r \theta}_{p}(z) r$. For a more exact description of the angular spread in Gaussian beams, the effective radiation source should be used: $s^{\text {eff }}=\overline{r \theta}_{p}(z) / \bar{\theta}_{p}^{2}(z)$ as discussed in more detail elsewhere [20].

The energy loss related to the distribution function [Eq. (14)] thus enters the equation via the energy dependence of the mass-scattering power, $T_{p}[\bar{E}(u)]$. The function $h(z, r, \theta)$ is thus identical to the analytical pencil beam solution often used for description of the electron and ion transport in narrow beams. Such a transport equation is based on the assumption that the primary particles are not lost from the beam. Such losses are taken into account in the present transport equation as described by the longitudinal transport function $g$ discussed in more detail in the next section.

\section{The solution of longitudinal transport $g_{p}(z)$}

Under the CSDA assumption the simpler transport equation for $g$ is given by Eq. (9). We introduce the variable transformation $z=R_{0}(1-t), d z=-R_{0} d t$ based on the fact that for $z=0, E=E_{0}$ and $t\left(E_{0}\right)=R\left(E_{0}\right) / R\left(E_{0}\right)=1$, cf. Eq. (7). In addition, for convenience, a redefinition of the function $g$ is introduced so that $g(1-z) \rightarrow g(z)$. The transport equation [Eq. (9)] then results in the relation between differentials:

$$
\frac{d g_{p}(z)}{g_{p}(z)}=\mu(z) d z .
$$


By direct integration, Eq. (21) results in the simple solution:

$$
g_{p}(z)=g_{p 0} \exp \left(-\int_{0}^{z} \mu(E(u)) d u\right)=g_{p 0} \exp (-\bar{\mu}(z) z),
$$

where $g_{p}(z)$ is the total number of remaining primary ions at penetration depth $z, g_{p 0}$ is the number of incident primaries at $z=0$, and $\bar{\mu}(z)$ is the longitudinal transport integral corresponding to the lateral transport integrals $\bar{r}_{p}^{2}(z), \bar{\theta}_{p}^{2}(z)$, and $\overline{r \theta}_{p}(z)$ in Eqs. (15)-(17).

The mean attenuation coefficient $\bar{\mu}_{p}(z)$ for loss of primary ions from the beam at energy $E$ and depth $z$ is defined by

$$
\bar{\mu}_{p}(z) \equiv \frac{\int_{0}^{z} \mu_{p}[E(u)] d u}{\int_{0}^{z} d u},
$$

where often the mean value over the therapeutic range can be used as a transport constant, according to $\bar{\mu}_{p}(z)=$ $\bar{\mu}_{p}\left(R_{p}\right) \approx \bar{\mu}_{p}[17,21-24]$.

\section{The total distribution function of the primary particles}

By combining the solutions, from the lateral and longitudinal equations [Eqs. (14) and (22)] the total fluence or phase space density of the primary particles $p$ is given by

$$
\begin{aligned}
\Phi_{p}(z, r, \theta)= & g(z) h(z, r, \theta) \\
= & g_{p 0} \exp \left[-\bar{\mu}_{p}(z) \cdot z\right] \frac{\exp \left(-\frac{r^{2}}{\bar{r}_{p}^{2}(z)}\right)}{\pi \bar{r}_{p}^{2}(z)} \\
& \times \frac{\exp \left(-\frac{[\theta-\theta(r)]^{2}}{\bar{\theta}_{p, c}^{2}(z)}\right)}{\pi \bar{\theta}_{p, c}^{2}(z)},
\end{aligned}
$$

where $g_{p 0}=\Phi_{p}(0,0,0) \pi^{2} \bar{r}_{p}^{2}(0) \bar{\theta}_{p, c}^{2}(0)$.

Furthermore, by integration of Eq. (24) over all radii, we obtain the purely angular distribution for an infinitely wide beam:

$$
\Phi_{p}(z, \theta)=\Phi_{p}(0,0)^{2} \bar{\theta}_{p}^{2}(0) \exp \left[-\bar{\mu}_{p}(z) z\right] \frac{\exp \left(-\frac{\theta^{2}}{\bar{\theta}_{p}^{2}(z)}\right)}{\bar{\theta}_{p}^{2}(z)},
$$

where $\bar{\theta}_{p}^{2}(z)$ can be calculated based on the mean energy and depth dependence of the primary particles, cf. Eq. (17) and [17]. Similar to Eq. (25), the purely spatial distribution is given by integrating Eq. (24) over all angles,

$$
\Phi_{p}(z, r)=\Phi_{p}(0,0)^{2} \bar{r}_{p}^{2}(0) \exp \left[-\bar{\mu}_{p}(z) z\right] \frac{\exp \left(-\frac{r^{2}}{\bar{r}_{p}^{2}(z)}\right)}{\bar{r}_{p}^{2}(z)},
$$

where $\bar{r}_{p}^{2}(z)$ can be derived from Eq. (15), based on the energy dependent mass-scattering power. The longitudinal distribution inside a radial interval, $r \leq R$, can be derived by simple integration of Eq. (26), with the solution

$$
\begin{aligned}
N_{p}(z, R)= & \Phi_{p}(0,0) \pi \bar{r}_{p}^{2}(0) \int_{0}^{R} \exp \left[-\bar{\mu}_{p}(z) z\right] \\
& \times \frac{\exp \left(-\frac{r^{2}}{\bar{r}_{p}^{2}(z)}\right)}{\pi \bar{r}_{p}^{2}(z)} 2 \pi r d r=\Phi_{p}(0,0) \pi \bar{r}_{p}^{2}(0) \\
& \times \exp \left[-\bar{\mu}_{p}(z) z\right]\left[1-\exp \left(-\frac{R^{2}}{r_{p}^{2}(z)}\right)\right] .
\end{aligned}
$$

Here, Eq. (27) implies that the number of ions inside a radii $R$ from the beam axis, when $\bar{\mu}_{p}(z) \approx \mu_{p}$, is characterized by a purely exponential attenuation at depth for which $\bar{r}_{p}^{2}(z) \ll R^{2}$. As the value of $\bar{r}_{p}^{2}(z)$ increases with penetration depths, a stronger decrease of the fluence is expected due to the enhanced lateral spread of the beam. The fluence of primary ions in a broad beam can be calculated from the pencil beam distribution function [Eq. (24)]. The fluence of a broad uniform beam is thus obtained by integrating Eq. (24) over all radius and angles and the practical range $R_{p}$ :

$$
\Phi_{p}(z)=\Phi_{p}(0) \exp \left[-\bar{\mu}_{p}(z) z\right]
$$

where $\bar{\mu}_{p}(z)$ in Eq. (28) can be rather accurately approximated by $\bar{\mu}_{p}\left(R_{p}\right) \approx \bar{\mu}_{p}$, [Eq. (23) and [16,17] ]; that is, the mean value over the whole practical range. Furthermore, Eq. (28) is valid approximately up to the practical range $R_{p}$ of the particle where the fluence is rapidly reduced to zero. The practical range can be derived analytically based on knowledge about the initial particle energy [17].

The quantity $\Phi_{0} \exp \left(-\bar{\mu}_{p} z\right)$ is thus a measure of the remaining primary ion fluence and thus the number of primary particles at the depth $z$, where a higher value of the macroscopic cross section, $\bar{\sigma}_{a}$, and a shorter mean-free path is closely associated with increasing atomic charge and weight of the projectile. According to the above phase space density function [Eq. (24)] the beam will thus remain exponentially attenuated, with an energy dependent spatial spread since for most cases $\bar{\mu}_{p} \approx \bar{\mu}_{p}\left(R_{p}\right)$. Because the continuous slowing down approximation is taken into account here, there is a very well-defined relation between the average energy loss, the lateral displacement, the longitudinal range straggling, and the penetration depth for the primary ions.

\section{Absorbed dose of primary particles in a pencil beam}

The local absorbed dose of the primary particles in a pencil beam can be given by integration of the primary fluence, $\Phi_{E}^{P}(z, r)$, Eq. (26) multiplied by the associated stopping power, cf. [2]: 


$$
\begin{aligned}
D^{P}(z, r) & =\int_{0}^{E_{\max }} \frac{S^{p}(E)}{\rho} \Phi_{E}^{p}(z, r) d E \\
& =\frac{\bar{S}^{\Phi, p}(z)}{\rho} \Phi^{p}(z, r) \approx \frac{S^{p}\left[\bar{E}^{p}(z)\right]}{\rho} \Phi_{p}(z, r),
\end{aligned}
$$

where $\rho$ is the density and $\bar{S}^{\Phi, p}(z)$ the fluence weighted mean stopping power, which in the first approximation can be derived from the depth dependence of the mean energy $\bar{E}^{p}(z)$ [17]. Higher order corrections of the fluence and stopping power can be derived by taking into account the increasing range straggling towards the Bragg peak.

\section{B. Transport of fragments set in motion by the primaries}

The distribution function of fragments generated in a high-energy light ion pencil beam can be derived from the presented solution of the primary particle transport. In the first approximation, the fragments are assumed to be produced according to the primary particles absorption cross section given by $\bar{\mu}(z) \approx \bar{\mu}$. The generated fluence of the fragments can thus be attributed to the inelastic nuclear interactions of the primary particles. The amount of the generated fragments' fluence will therefore depend on the macroscopic cross section of these nuclear interactions. From the solution of the transport of the primary ions, [Eq. (24)], loss of primaries correlated with generation of fragments can occur at any depth, independent of the lateral position. The depth dependence of the fluence of fragments generated in a pencil beam of primary high-energy light ions can in a first approximation be assumed to have an energy independent cross section and simple exponential fluence attenuation as similar to that of the primaries. In addition, the depth dependence of the fragments depends on the energy-range relation of the individual fragments relative to that of the primary projectiles, cf. [16]. Also, the momentum distribution of the fragments is likely to be quasi-Gaussian in the rest frame of the projectile with a quite narrow angular distribution [25-27]. This approximation holds best for fragments of charge $z \geq 2$ [16]. The accumulated fluence of fragments of type $i, \Phi_{i}(z, r, \theta)$, may therefore be expressed according to

$$
\begin{aligned}
\Phi_{i}(z, r, \theta)= & -\int_{0}^{\min \left(z, R_{p}\right)} \\
& \times C_{i} \frac{d \Phi_{p}(s)}{d s} e^{-\mu_{i}(z-s)} \bar{r}_{i}^{2}(0,0) \bar{\theta}_{i, c}^{2}(0,0) \\
& \times \frac{e^{-\left[r^{2} / \bar{r}_{i}^{2}(z, s)\right]}}{\bar{r}_{i}^{2}(z, s)} \frac{e^{-\left\{\left[\left(\theta-\theta_{i, r}\right)^{2}\right] /\right\} \bar{\theta}_{i, c}^{2}(z, s)}}{\bar{\theta}_{i, c}^{2}(z, s)} d s .
\end{aligned}
$$

The minus sign in Eq. (30) accounts for the fact that a primary fluence loss along the track, $d s$, gives a positive secondary particle fluence contribution. This expression characterizes the accumulated fluence of fragment $i$ in the region in front of and beyond the practical range, $R_{p}$, of the primary particles, taking into account that energy and fluence contributions will be zero, beyond the range of the fragments or when $z>s+R_{i 0}(s)$. The secondaries of type $i$ produced at $s$ are assumed to be exponentially absorbed to depth $z$ by the attenuation coefficient $\mu_{i}$. $R_{i 0}(s)$ is the continuous slowing down range of the fragments, set in motion at depth $s$, cf. Ref. [16].

The fraction of each type of produced fragment $i$ is given by $C_{i}$. At depth $z$, the mean square radius $\bar{r}^{2}(z, s)$ of the fragments set in motion at $(s<z)$ is given by

$$
\begin{aligned}
\bar{r}_{i}^{2}(z, s)= & \bar{r}_{p}^{2}(s)+\left[2 \overline{r \theta}_{p}(s)\right](z-s)+\left[\bar{\theta}_{p}^{2}(s)+\bar{\theta}_{i}^{2}(s)\right] \\
& \times(z-s)^{2}+\int_{s}^{z}(z-u)^{2} T_{i}\left[E_{i}(u)\right] d u,
\end{aligned}
$$

where $\bar{r}_{p}^{2}(s)$ is the mean square radius of the primary particles at the position $s$ along the $z$ axis where the secondaries are generated. Here, $\bar{r}_{i}^{2}(s) \equiv \bar{r}_{p}^{2}(s)$ and $\overline{r \theta}_{i}(s) \equiv \overline{r \theta}_{p}(s)$, since the transport of these fragments, in the first approximation, is initiated by the primary particles at position $s$ in the beam. The $\bar{\theta}_{i}^{2}(s)$ is the additional initial mean square angular spread of the fragments as they are generated at the position $s$ along the $z$ axis and is of the order of a few degrees [28-30]. $T_{i}\left(E_{i}(u)\right)$ is the depthdependent scattering power of the fragments at the position $u$ along the $z$ axis, based on the energy depth dependent and continuous slowing down range of the fragments [16], taking into account that energy and fluence contributions will be zero beyond the range of the fragments or when $z>$ $s+R_{i 0}(s)$. In analogy with Eq. (31), the covariance $\overline{r \theta}_{i}(z, s)$ of the fragments describing the increase in the most probable inclination of the fragments off axis is given by

$$
\begin{gathered}
\overline{r \theta}_{i}(z, s)=\overline{r \theta}_{p}(s)+\left[\bar{\theta}_{p}^{2}(s)+\bar{\theta}_{i}^{2}(s)\right](z-s) \\
+\int_{s}^{z}(z-u) T_{i}\left[E_{i}(u)\right] d u,
\end{gathered}
$$

where $\overline{r \theta}_{p}(s)$ is the covariance of the primary particles at the depth $s$ and is equal to the initial covariance of the fragments as they are generated at the depth $s$.

The mean angular spread $\bar{\theta}_{i}^{2}(z, s)$ of the fragments at depth $z$ is similarly given by

$$
\bar{\theta}_{i}^{2}(z, s)=\bar{\theta}_{p}^{2}(s)+\bar{\theta}_{i}^{2}(s)+\int_{s}^{z} T_{i}\left[E_{i}(u)\right] d u,
$$

where $\bar{\theta}_{p}^{2}(s)$ is the mean square angular spread of the primary particles in the beam at depth $s$. The fragments' energy loss related to the distribution function [Eq. (30)] thus enters the equation, similar to the primary transport, via the energy dependence of the mass-scattering power, $T_{i}\left[E_{i}(u)\right]$. The mean square angular spread of the fragments at any radial distance $r$ in the beam is independent on $r$ in the present approximation, $\bar{\theta}_{i, c}^{2}(z, s)$, and is given by 


$$
\bar{\theta}_{i, c}^{2}(z, s)=\bar{\theta}_{i}^{2}(z, s)-\frac{\left[\overline{r \theta}_{i}(z, s)\right]^{2}}{\bar{r}_{i}^{2}(z, s)} .
$$

By inserting the expressions for the primary particles from above [Eqs. (15)-(17)] and neglecting differences between individual energy and range relations of the primary particle and the generated fragment, the radial spread [Eq. (31)] of the fragments can be reduced to

$$
\begin{aligned}
\bar{r}_{i}^{2}(z, s)= & \bar{r}_{p}^{2}(z)+\bar{\theta}_{i}^{2}(s)(z-s)^{2}+\int_{s}^{z}(z-u)^{2} \\
& \times\left[T_{i}(u)-T_{p}(u)\right] d u,
\end{aligned}
$$

where $T_{p}(u)$ is the mass-scattering value of the primary particle. Interestingly, the radial spread of the secondaries is equal to that of the primaries plus the correction for the angular spread of the production process and a term depending on the difference in scattering power between primaries and fragments. In analogy to the radial spread, Eqs. (32) and (33) reduce to

$$
\begin{aligned}
\overline{r \theta}_{i}(z, s)= & \overline{r \theta}_{p}(z)+\bar{\theta}_{i}^{2}(s)(z-s) \\
& +\int_{s}^{z}(z-u)\left[T_{i}(u)-T_{p}(u)\right] d u
\end{aligned}
$$

and

$$
\bar{\theta}_{i}^{2}(z, s)=\bar{\theta}_{p}^{2}(z)+\bar{\theta}_{i}^{2}(s)+\int_{s}^{z}\left[T_{i}(u)-T_{p}(u)\right] d u .
$$

The rewritten expressions [Eqs. (35)-(37)] are, as mentioned, based on simplified particle transport assumptions and will therefore be discussed no further here. The most probable angle of motion of the fragments at a radial distance $r$ is given by

$$
\theta(r)_{i}=\frac{\overline{r \theta}_{i}(z, s)}{\bar{r}_{i}^{2}(z, s)} r=\frac{r}{s_{i, \mathrm{vir}}},
$$

where $s_{i, \text { vir }}$ is the distance to the individual virtual point source of the fragments $i$, generated at depth $s$. Similar to the primary ion transport, the purely angular distribution in an infinitely wide beam is obtained when integrating Eq. (30) over all radii

$$
\begin{aligned}
\Phi_{i}(z, \theta)= & -\int_{0}^{z} C_{i} \bar{\theta}_{i}^{2}(0,0) \\
& \times \frac{d \Phi_{p}(s)}{d s} e^{-\mu_{i}(z-s)} \frac{e^{-\left[\theta^{2} / \bar{\theta}_{i}^{2}(z, s)\right]}}{\bar{\theta}_{i}^{2}(z, s)} d s,
\end{aligned}
$$

where $\bar{\theta}_{i}^{2}(z, s)$ can be derived from the energy and depth dependence and mass-scattering power expression of the fragments [Eq. (33) and [16] ].

Also, the pure spatial distribution of the secondaries is obtained by integrating Eq. (30) over all angles,

$$
\begin{aligned}
\Phi_{i}(z, r)= & -\int_{0}^{z} C_{i} \bar{r}_{i}^{2}(0,0) \\
& \times \frac{d \Phi_{p}(s)}{d s} e^{-\mu_{i}(z-s)} \frac{e^{-\left[r^{2} / \pi \bar{r}_{i}^{2}(z, s)\right]}}{\bar{r}_{i}^{2}(z, s)} d s .
\end{aligned}
$$

The spatial distribution inside a cylinder or radial interval, from $0 \rightarrow R$, along the $z$ axis can be derived by integrating Eq. (40):

$$
\begin{aligned}
N_{i}(z, R)= & \int_{0}^{z} \int_{0}^{R} C_{i} \pi \bar{r}_{i}^{2}(0,0) \\
& \times \frac{d \Phi_{p}(s)}{d s} e^{-\mu_{i}(z-s)} \frac{e^{\left[-r^{2} / r_{i}^{2}(z, s)\right]}}{\pi r_{i}^{2}(z, s)} 2 \pi r d r d s \\
= & \int_{0}^{z} C_{i} \pi \bar{r}_{i}^{2}(0,0) \\
& \times \frac{d \Phi_{p}(s)}{d s} e^{-\mu_{i}(z-s)}\left(1-e^{\left[-R^{2} / r_{i}^{2}(z, s)\right]}\right) d s .
\end{aligned}
$$

The buildup and spatial distribution function of fragments at penetration depths for which $\bar{r}_{i}^{2}(z, s) \ll R^{2}$ will thus result in an expression identical to that for the fluence of fragments generated in a broad beam unless the factor $\pi \bar{r}_{i}^{2}(0,0)$. From Eq. (41) it is also easy to realize that the relative amount of fragments in a region close to the central axis will decrease with increasing depth for $R^{2} \ll \bar{r}_{i}^{2}(z, s)$, where an increasing value of the mean square radius of the fragments $\bar{r}_{i}^{2}(z, s)$ along the central axis is a direct consequence of the fragments internal lateral spread conditions related to the values $\bar{r}_{p}^{2}(s), \overline{r \theta}_{p}(s), \bar{\theta}_{p}^{2}(s), \bar{\theta}_{i}^{2}(s)$, and $T_{i}\left[E_{i}(u)\right]$.

The fluence of the fragments in a broad beam can be calculated from the pencil beam distribution function [Eq. (30)]. The total fluence for the broad beam is thus obtained by integrating Eq. (30) over all radii and angles:

$$
\Phi_{i}(z)=-\int_{0}^{\min \left(z, R_{p}\right)} C_{i} \frac{d \Phi_{p}(s)}{d s} e^{-\mu_{i}(z-s)} d s .
$$

The integrals of the fluence expressions for fragments can be evaluated accurately as previously shown in [16]. The absorbed dose of the heavy fragments in a pencil beam can be obtained to first approximation from the energy and fluence depth dependence, similar to the transport of primary particles, and could then be approximated by

$$
D_{i}(z, r)=\bar{S}_{i}(z) \Phi_{i}(z, r) \approx \frac{\bar{S}_{i}\left[\bar{E}_{i}(z)\right]}{\rho} \Phi_{i}(z, r),
$$

where the fluence weighted mean stopping power, $\bar{S}_{i}(z) \approx$ $\bar{S}_{i}\left[\bar{E}_{i}(z)\right]$, is based on the depth dependence of the fragments' mean energy, and the radial fluence depth dependence $\Phi_{i}(z, r)$ accounts for the individual ranges and corresponding lateral spread of the fragments. The light fragments pencil beam absorbed dose is more simply formulated in terms of the depth dependence of the fluence taking the quasiconstant stopping power into account according to 


$$
D_{i}(z, r)=\frac{\bar{S}_{i}(z)}{\rho} \Phi_{i}(z, r) \approx \frac{\bar{S}_{i}}{\rho} \Phi_{i}(z, r),
$$

where $\bar{S}_{i}$ is the almost constant mean stopping power of low LET, quasi-independent of depth and energy.

\section{MONTE CARLO CALCULATIONS}

The Monte Carlo code SHIELD-HIT $[19,20]$ as well as the modified version SHIELD-HIT $+[16,17]$ were used to calculate the fluence and absorbed dose for broad and narrow ${ }^{7} \mathrm{Li},{ }^{11} \mathrm{C}$, and ${ }^{12} \mathrm{C}$ pencil beams. The code SHIELD-HIT simulates the interactions of hadrons and atomic nuclei of arbitrary charge and mass number with variable targets in the energy range from $1 \mathrm{TeV} / \mathrm{u}$ down to $25 \mathrm{keV} / \mathrm{u}$. Multiple Coulomb scattering and energy loss straggling are taken into account. The production of fragments due to inelastic nuclear interactions was simulated in SHIELD-HIT using the multistage dynamical nucleus models where all types of hadron-nucleus and nucleus-nucleus inelastic interactions are taken into account [31]. A refined version of the Monte Carlo code SHIELD-HIT, described in Refs. [16,17], has recently been developed in order to calculate a wide range of radiation field quantities. In this version SHIELD-HIT ${ }_{+}$, the fluence differential in both energy and angle was determined both for the primary particles and their fragments. Calculations have been performed for a point monodirectional and monoenergetic ion beam of perpendicular incidence at the center of a cylindrical water phantom $(R=10 \mathrm{~cm}, L=50 \mathrm{~cm})$. The fluence differential in energy and angle was scored separately in cylindrical rings of a thickness of $1 \mathrm{~mm}$ and diameters from $1 \mathrm{~mm}$ up to $20 \mathrm{~cm}$. The energies of the projectiles were chosen to correspond to ranges in water of approximately 150 and $260 \mathrm{~mm}$. The stopping power of primary ${ }^{7} \mathrm{Li},{ }^{11} \mathrm{C}$, and ${ }^{12} \mathrm{C}$ and all fragments in water have been calculated using ICRU-49 [32] and ICRU-73 [33] recommended data. The value of the attenuation coefficient of primaries, $\mu_{p}$, was determined for simplicity by least square fit to SHIELD-HIT Monte Carlo data. Table I lists the important physical, numerical, and statistical parameters related to the simulations. The consistency of SHIELD-HIT and SHIELD-HIT + with experimental data

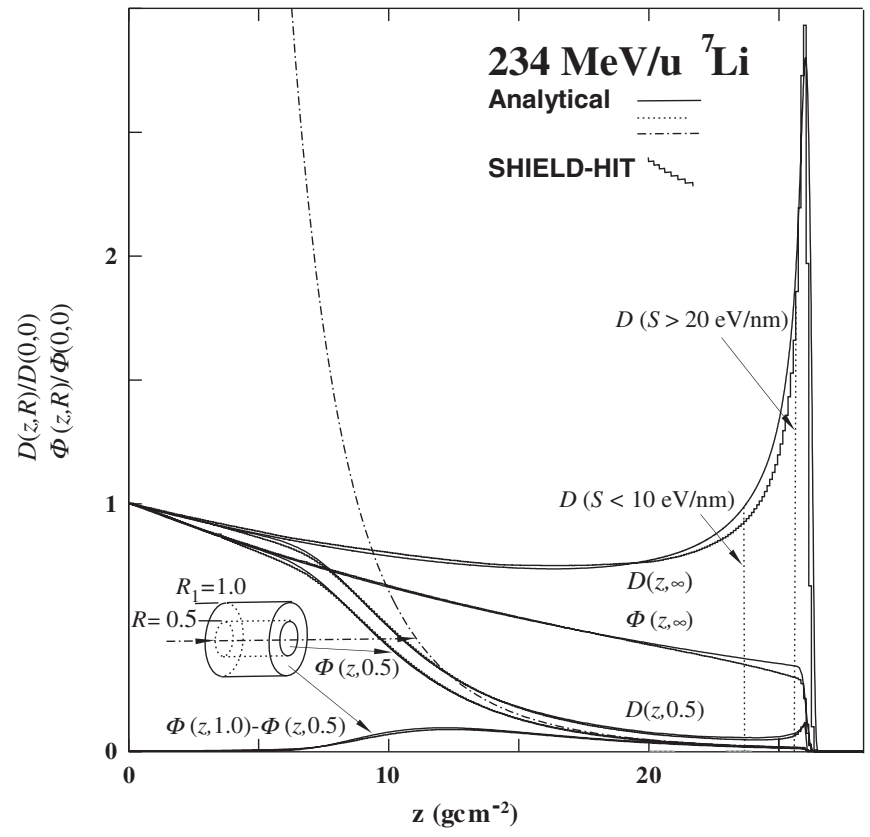

FIG. 1. Comparison of analytical (solid curves) and MC (histogram curves) depth variation of the broad beam, $\Phi(z)$ [Eq. (28)] and the fluence incident of cylindrical targets $\Phi(z,|r| \leq R)$ [Eq. (27)] as well as the broad beam absorbed dose, $D(z)$ [17] and the narrow beam absorbed dose $D(z, R)$ [Eq. (29)] of primary ${ }^{7} \mathrm{Li}$ ions in a $234 \mathrm{MeV} / \mathrm{u}$ beam in water. The dotted lines are the primary absorbed dose, analytically calculated, divided into its low $(<10 \mathrm{eV} / \mathrm{nm})$ and high $(>20 \mathrm{eV} / \mathrm{nm})$ LET components. The results have been normalized to the primary values at the phantom surface. The point monodirectional pencil beam data are evaluated inside cylindrical shells with radii of $0-0.5 \mathrm{~mm}$ and $0.5-1.0 \mathrm{~mm}$. The loss of primaries and the enhanced scattering events result in a significant reduction of the fluence and absorbed dose value close to the central axis. Interestingly, the true central axis fluence is considerably higher as indicated by the dashdotted lines reaching a value about 25 at the surface.

and other Monte Carlo codes has been verified earlier by comparing depth energy deposition profiles and other physical beam parameters of light ions $[2,16,18,19,27,34-36]$.

TABLE I. The physical, numerical, and statistical parameters related to the simulations. The simulations take into account Gaussian range straggling, multiple scattering, and nuclear reactions. The relative standard deviations, $\sigma_{\phi_{1}} / \phi_{1}$ and $\sigma_{\phi_{2}} / \phi_{2}$, correspond to the statistical uncertainties of the calculated fluence in the simulation. Type 1 here relates to the relative standard deviation in the plateau region and type 2 in the Bragg peak region.

\begin{tabular}{lcccccc}
\hline \hline $\begin{array}{l}\text { Quantity } \\
\text { Unit }\end{array}$ & Particle & $\begin{array}{c}\text { Energy } \\
\mathrm{MeV} / \mathrm{u}\end{array}$ & $\begin{array}{c}\text { Cutoff } \\
\mathrm{keV} / \mathrm{u}\end{array}$ & Number of histories & $\begin{array}{c}\sigma_{\Phi_{1}} / \Phi_{1} \\
\%\end{array}$ & $\begin{array}{c}\sigma_{\Phi_{2}} / \Phi_{2} \\
\%\end{array}$ \\
\hline & 234 & 25 & $10^{6}$ & $<0.1^{\mathrm{a}}$ & $0.2^{\mathrm{a}}$ \\
& ${ }^{7} \mathrm{Li}$ & 288 & 25 & $10^{6}$ & $\approx<0.1$ & $\approx 0.2$ \\
& ${ }^{11} \mathrm{C}$ & 279 & 25 & $10^{6}$ & $\approx<0.1$ & $\approx 0.2$ \\
\hline \hline
\end{tabular}

${ }^{\mathrm{a}}$ Personal communication with Martha Hultqvist. 
In therapeutic light ion beams such as Li, the greater part of the dose and practically all the high LET dose components are delivered by the primary particles [2]. From a biologically optimized treatment planning point of view, an accurate description of the primary ion transport in such beams is therefore highly desirable. The clinically useful results in use of ${ }^{12} \mathrm{C}$ ions since the beginning of the 1990's, at the clinical research facility, HIMAC in Chiba, Japan [1] and later at Darmstadt, GSI, have led to the design of and plans for additional facilities both in Japan and Europe. For the successful application of beams, such as ${ }^{12} \mathrm{C}$, an accurate description of the transport of its associated fragments, such as ${ }^{11} \mathrm{~B}$ and ${ }^{11} \mathrm{C}$, is highly important. This helps to accurately localize the high LET regions, where the highest variations in the relative biological effectiveness (RBE) are expected as well as the position of generated positron emitters. Furthermore, the positron emitting radioactive beam in itself, such as ${ }^{11} \mathrm{C}$, is of high therapeutic interest, not least due to improvements in treatment verification using in vivo PET imaging. For this reason, the primary light ions ${ }^{7} \mathrm{Li},{ }^{11} \mathrm{C}$, and ${ }^{12} \mathrm{C}$ and associated fragments, ${ }^{11} \mathrm{C}$

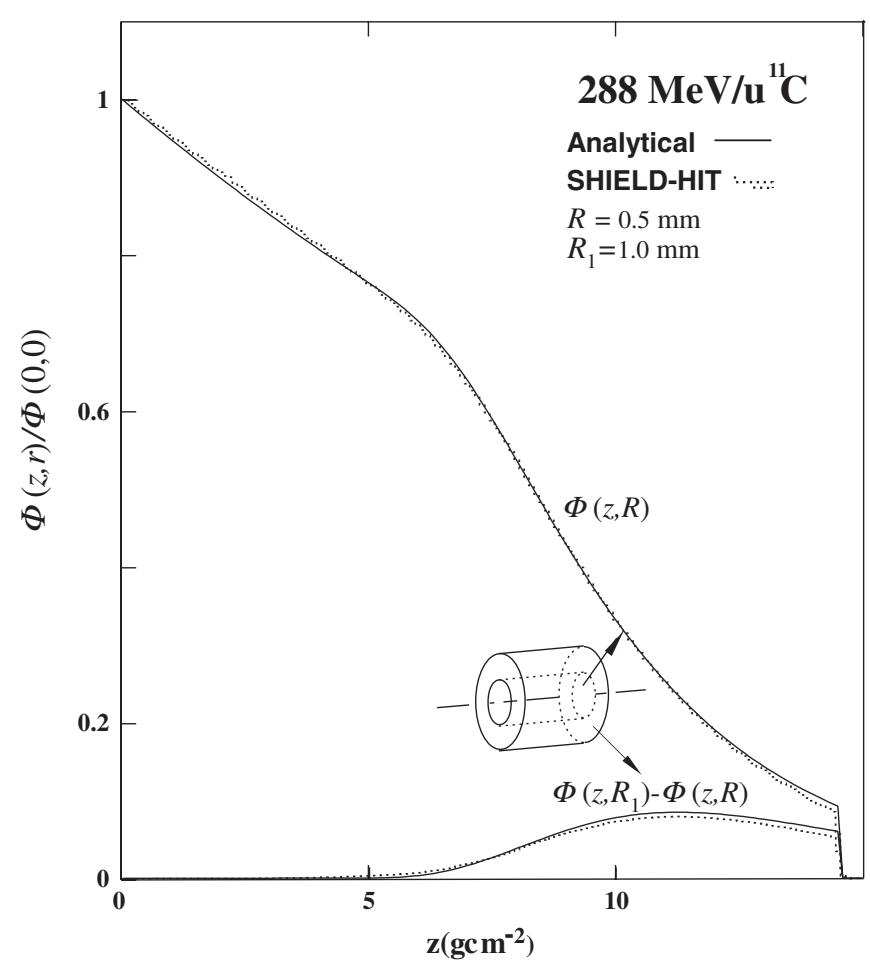

FIG. 2. The depth variation of the analytical narrow depth fluence (solid lines) $\Phi(z, R)$ and $\Phi\left(z, R_{1}\right)$ [Eq. (27)], and the $\mathrm{MC}$ calculated fluences (dotted histograms) for the primary ${ }^{11} \mathrm{C}$ $\beta^{+}$emitter. The shape of the pencil beam depth fluence curve coincides with shape of the broad beam depth fluence, up to the range of $5 \mathrm{~cm}$ owing to the low mass-scattering power value at high energies of the projectile. The close agreement between the analytical calculation and the MC data confirms the present pencil beam solution of the Boltzmann equation for the primary ions. and ${ }^{11} \mathrm{~B}$, were used to illustrate the present theory versus Monte Carlo through the paper.

\section{RESULTS}

In Figs. 1-3, the analytical depth variation of the primary ${ }^{7} \mathrm{Li},{ }^{11} \mathrm{C}$, and ${ }^{12} \mathrm{C}$ ions fluence [Eqs. (27) and (28)] and absorbed dose [Eq. (29)] in a narrow and broad beam (solid line) are compared with Monte Carlo simulations (solid and dotted histograms). The curves have been normalized to the respective broad and narrow beam values at the phantom surface, $z=0$.

The analytical solutions for the narrow and broad beam fluence and absorbed dose are in close agreement with the MC results, where the analytical identification of the radiation quality in terms of low and high LET dose shows that the plateau region of the primary particles in a ${ }^{7} \mathrm{Li}$ beam of energy $234 \mathrm{MeV} / \mathrm{u}$ is comprised of mainly low LET $(<10 \mathrm{eV} / \mathrm{nm})$ components. The high LET $(>20 \mathrm{eV} / \mathrm{nm})$ components appear only in the Bragg peak characterized by a Gaussian distribution.

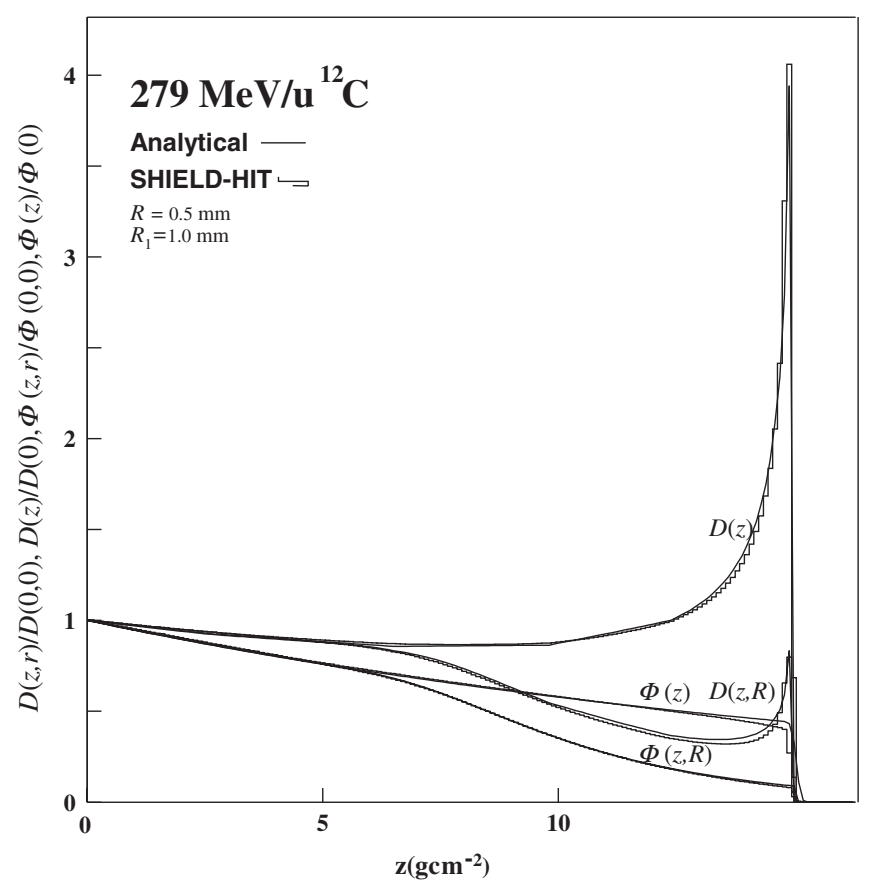

FIG. 3. Depth variation of the primary ${ }^{12} \mathrm{C}$ ions in broad and pencil beam in water described by analytical calculation of the absorbed dose and fluence [Eqs. (27)-(29)]. The histograms are SHIELD-HIT Monte Carlo for comparison. The reduced range straggling and higher stopping power of the lower energy ${ }^{12} \mathrm{C}$ beam result in a higher Bragg peak dose close to the central axis relative to the primary ${ }^{7} \mathrm{Li}$ ions, cf. Fig. 1. The depth fluence curves for the narrow and broad beams follow the shape of the depth fluence curve in the plateau region owing to the small variation of the mean stopping power. Only at the very end of the primary particle range is the loss in fluence accompanied by a compensating increased energy loss towards the Bragg peak. 
Furthermore, the exponential attenuation of the primary fluence and its enhanced lateral spread with increasing penetration depth has an important influence on the results. As seen in Figs. 1-3, it is clear that, at the depth for which $\bar{r}_{p}^{2}(z) \ll R^{2}$, the shape of the pencil beam depth fluence distribution coincides with the shape of the broad beam depth fluence distribution owing to the low mass-scattering power value at high energies of the projectile. As the energy of the particle decreases, also the mass scattering increases which results in an increased mean square radial spread $\bar{r}_{p}^{2}(z)$. Thus, both the loss of particles and the enhanced scattering events reduce the fluence and absorbed dose value along the central axis at larger penetration depth. In general, the radial spread for all these primaries is small. As shown in Figs. 1-3 most of the fluence of the primary is located along the $z$ axis at very small radii. This is clearly seen in Fig. 1 within the large difference between the fluence at the central axis and the fluence related to the inner diameter $(1 \mathrm{~mm})$. Because the continuous slowing down approximation is taken into account here, there is a very well-defined relation between the average energy loss, the lateral displacement, the longitudinal range straggling, and the penetration depth for the primary ions, as also shown in Figs. 1-3. The analytical Gaussian radial fluence distribution [Eq. (26)] of primary ${ }^{7} \mathrm{Li}$ at two penetration depths is illustrated in Fig. 4. The

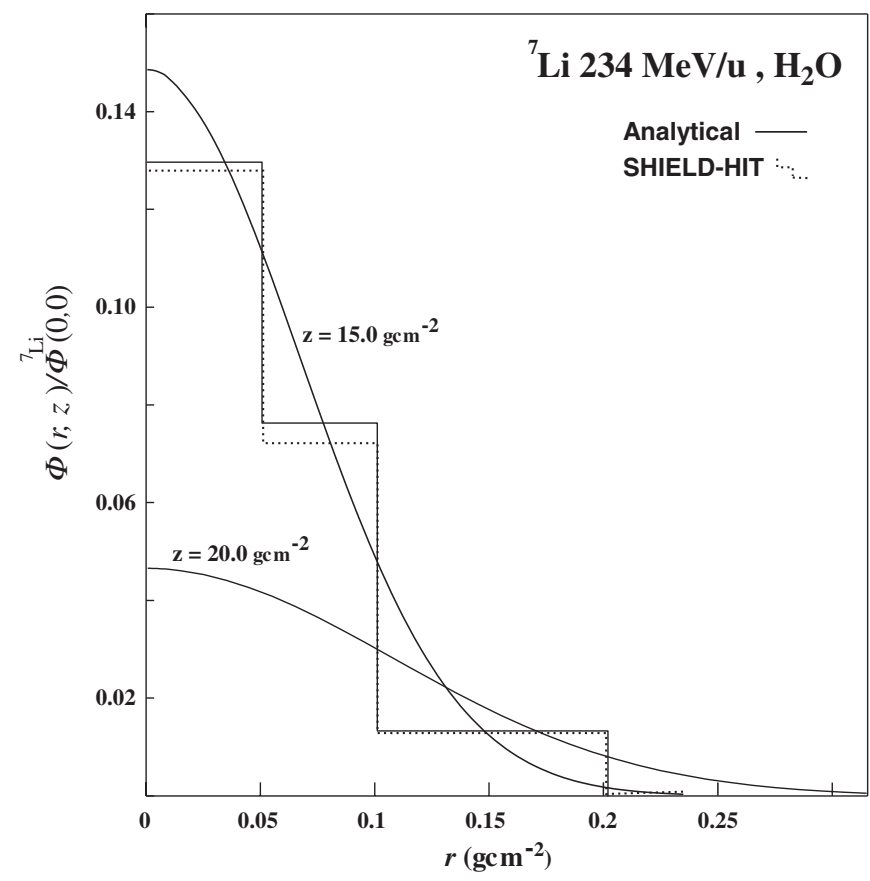

FIG. 4. The spatial analytical radial fluence distribution [Eq. (26)] at different depths (solid lines) of primary ${ }^{7} \mathrm{Li}$ in a $234 \mathrm{MeV} / \mathrm{u}{ }^{7} \mathrm{Li}$ beam in water compared with $\mathrm{MC}$ (dotted lines histograms). The results have been normalized to the primary particle values at the phantom surface. The advantages of the continuous analytical expressions developed in this paper are clearly seen here in relation to the segmental MC data. analytical results, solid lines, are compared with MC dotted histograms. The differences between the analytical calculations and the MC results for the primary fluence for each radial bin are, $1.2 \%, 5.4 \%$, and $2.3 \%$ when the scattering properties of the MC code are taken into account. The reduced fluence and the wider radial distribution are correlated with the attenuation and the reduced mean energy of the primary particles along the penetration depth. The advantage of the continuous analytical expressions is clearly seen in relation to the segmental MC data. In Fig. 5 the analytical depth variation of the pencil beam fluence (solid lines) of the ${ }^{11} \mathrm{C}$ fragments in a narrow ${ }^{12} \mathrm{C}$ beam are compared with $\mathrm{MC}$ (histograms). As predicted from Eq. (41), for these fragments, at shallow penetration depth, the fluence buildup in the pencil beam is closely related to the fluence buildup of the broad beam. Here the mean energy of the primary particles as well as the generated fragments is quite high. Therefore their respective mass-scattering values are low and hence also the value of the mean square radii, $\vec{r}_{i}^{2}(z, s)$ [Eq. (31)]. The fluence maxima of the generated fragments are strongly correlated

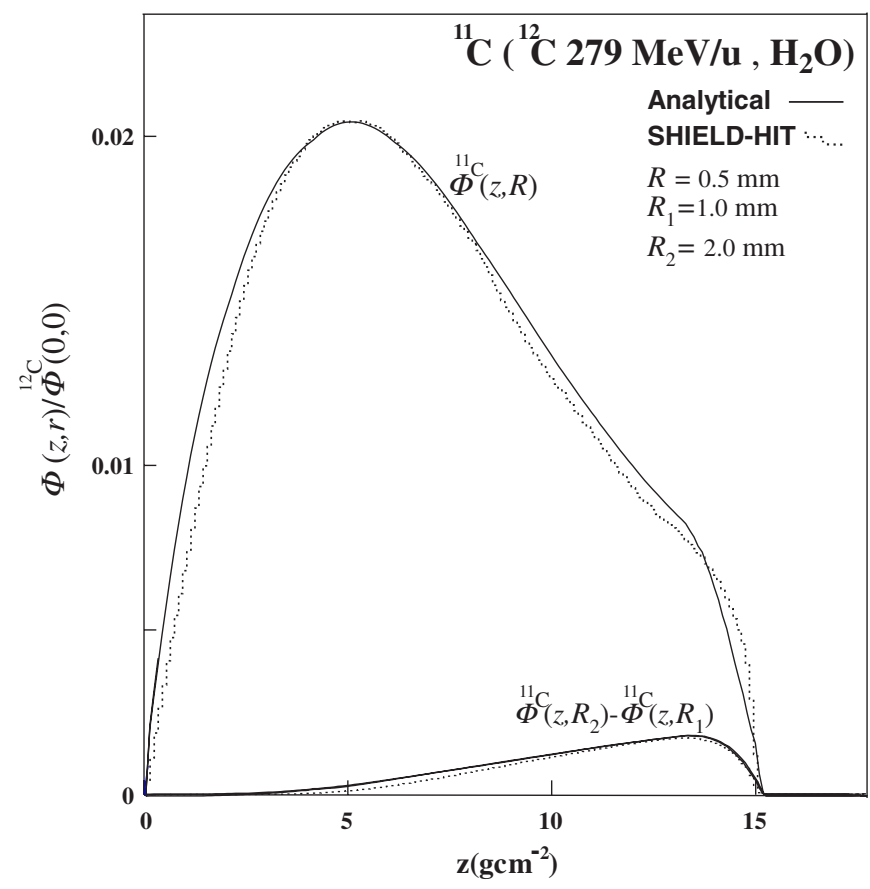

FIG. 5. The depth variation of the analytical narrow depth fluence, $\Phi(z, R), \Phi\left(z, R_{1}\right)$, and $\Phi\left(z, R_{2}\right)$ of the secondary ${ }^{11} \mathrm{C}$ $\beta^{+}$emitter in narrow $279 \mathrm{MeV} / \mathrm{u}{ }^{12} \mathrm{C}$ beam in water described by analytical calculation of the fluence [Eq. (41)] (solid curves). The dotted histograms are SHIELD-HIT Monte Carlo results for comparison. The shape of the ${ }^{11} \mathrm{C}$ fragments in the narrow ${ }^{12} \mathrm{C}$ beam is strongly influenced by a wider spatial spread of the primary particles, and the wider scattering process of the generated fragments, as the primary particle and the fragment energy decreases. Depending on the charge to mass ratio, the range of ${ }^{11} \mathrm{C}$ is slightly shorter relative the primary ion in contrast to the generated ${ }^{11} \mathrm{~B}$ ions, as previously discussed [16], cf. Figs. 6 and 7. 


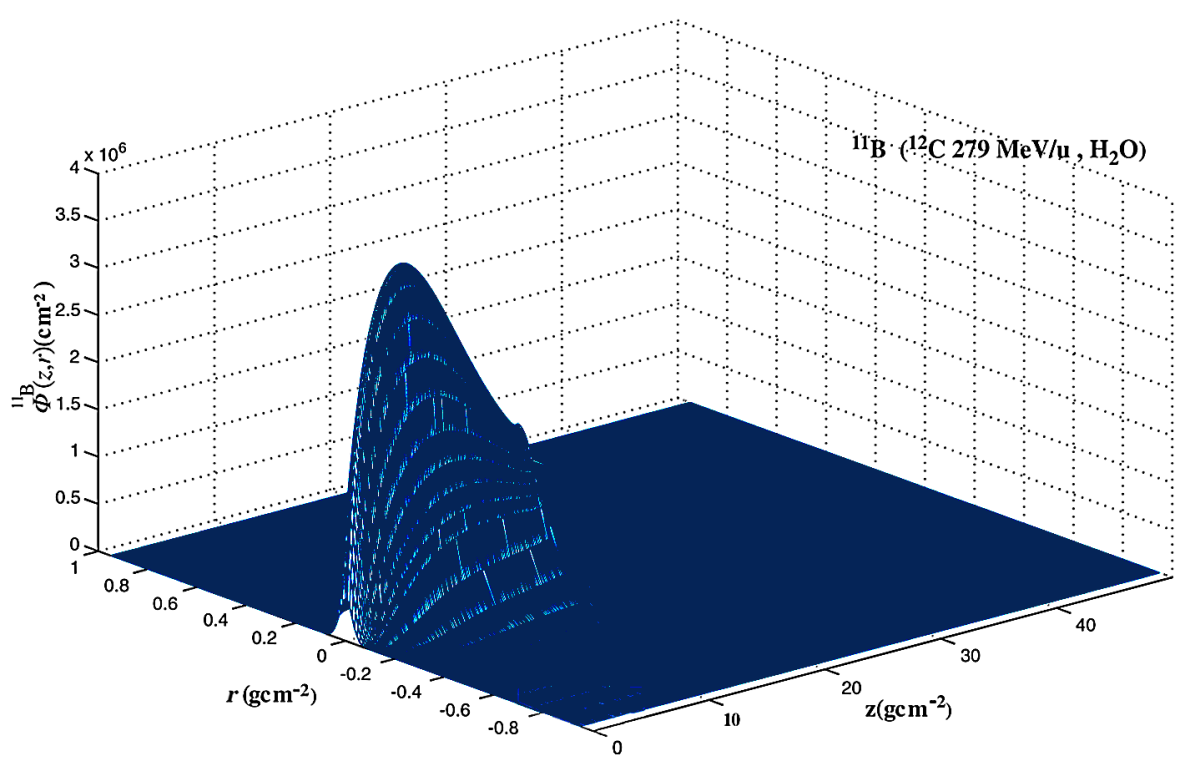

FIG. 6. The 3D distribution of the secondary ${ }^{11} \mathrm{~B}$ ion fluence in a $0.6 \mathrm{~mm}$ diameter incident ${ }^{12} \mathrm{C}$ beam in water, as described by the present analytical Eq. (40). The reduced fluence at larger penetration depth is strongly correlated with the wider lateral spread and sudden disappearance at the practical range of the primary particles and a wider lateral spread of the ${ }^{11} \mathrm{~B}$ fragments. The shape of the narrow depth fluence curve of the ${ }^{11} \mathrm{~B}$ and ${ }^{11} \mathrm{C}$ ions follows the shape of their broad beam depth fluence curve in the entrance region, since there, due to the high energy, the spatial spread of both the primary and the generated fragments is low, cf. Eq. (41). Since the range of the boron fragment is longer than the primary particle a secondary tail of $\Phi$ is seen.

with the region where an increase in the lateral displacement of the primary particles is expected and where the generated fragments have an increasing internal lateral spread. At further penetration depths, the analytical as well as the MC data show that the fluence close to the central axis falls much more steeply as a natural result of the increasing lateral displacement of both the primaries and the fragments as their mean energy is reduced and hence correlated with an increasing mass-scattering power value. The increasing lateral displacement at larger penetration depth of the generated fragments is also related to variation in the position at which the fragments are

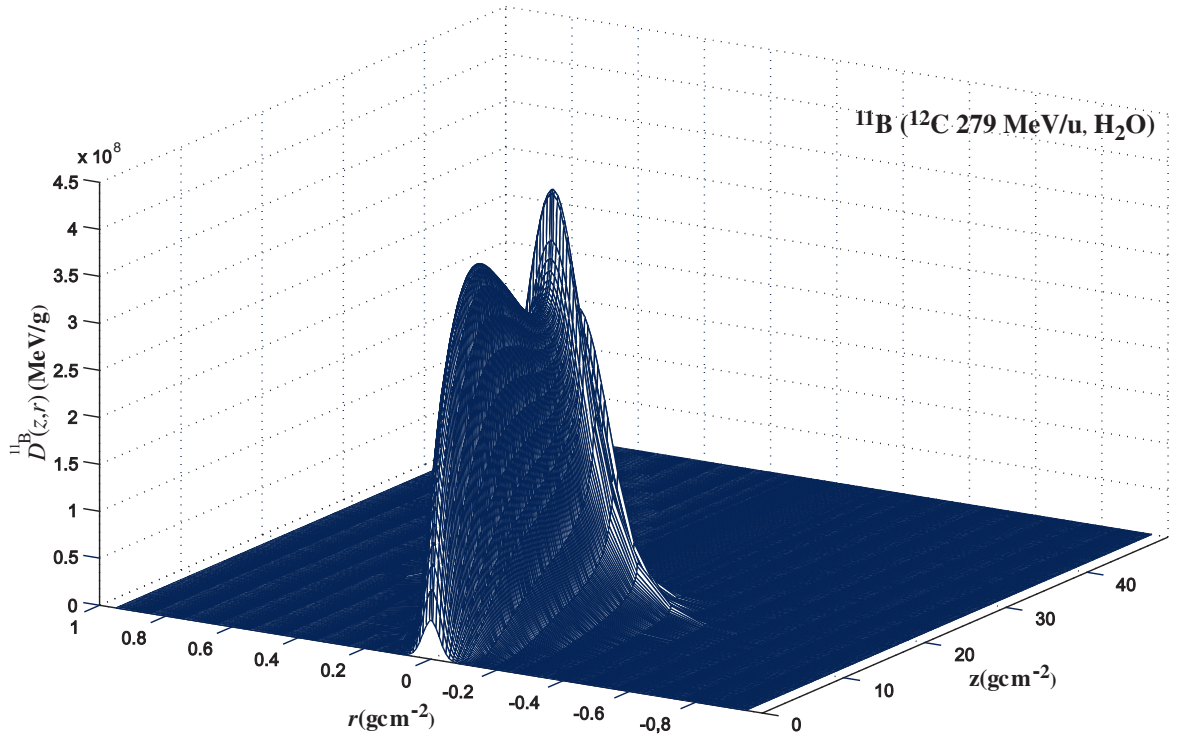

FIG. 7. The total analytical depth $3 \mathrm{D}$ absorbed dose variation [Eq. (43)] of secondary ${ }^{11} \mathrm{~B}$ ions in a $0.6 \mathrm{~mm}$ diameter ${ }^{12} \mathrm{C}$ beam in water (solid curves). The absorbed dose of these heavy fragments is clearly influenced by rapidly increasing energy loss towards the end of their ranges, cf. Fig. 6. An accurate 3D description of the transport of the heavier fragments, such as ${ }^{11} \mathrm{~B}$ and ${ }^{11} \mathrm{C}$ in the therapeutic ${ }^{12} \mathrm{C}$ beam, is of importance, since they help to accurately localize the high LET regions, where the greater variation in RBE are expected. 
produced from the primary particle as well as the increasing value of the initial angle of the fragments as they are set in motion at position $s$ along the $z$ axis. This wider lateral displacement is seen by the compensating fluence for the ${ }^{11} \mathrm{C}$ fragments at larger radii. The total 3D fluence distribution of the ${ }^{11} \mathrm{~B}$ fragments in a narrow ${ }^{12} \mathrm{C}$ beam [Eq. (40)] is illustrated in Fig. 6. The shape of the ${ }^{11} \mathrm{~B}$ fragments is quite well described by the influence of a wider lateral spread combined with a maximum range beyond the practical range of the primary particles. As with ${ }^{11} \mathrm{C}$ fragments, at shallow penetration depth, the fluence buildup of the ${ }^{11} \mathrm{~B}$ fragments is correlated with the fluence buildup of the broad beam [Eq. (41) and [16]]. In Fig. 7 the pencil beam absorbed dose [Eq. (43)] of the ${ }^{11} \mathrm{~B}$ fragments is also illustrated. In contrast to the 3D depth fluence distribution of the fragments, the absorbed dose will significantly rise at lower energies due to the increasing influence of stopping power as the energy decreases toward the end of the range of these fragments [16]. Since the energy distribution of these fragments is quite well defined, cf. [16], the associated low and high LET components can be identified analytically.

\section{DISCUSSIONS AND CONCLUSIONS}

A new solution of the Boltzmann equation considering the loss of primary particles and the transport of secondary fragments has been presented. The aim of the present solution is to describe the transport and to take into account the radiation quality distribution of light ions in the energy region relevant to radiation therapy. It is clear from the present solution of the Boltzmann equation that the exponential decrease of the fluence, the increased energy losses, and the effect of multiple scattering will determine the shape of the pencil and broad beam absorbed dose distributions of the primary projectiles. The importance of these effects generally increases as a function of penetration depth. Because of the high mean energy of the projectiles and their associated fragments in the entrance region, their mass-scattering power value is low and the shape of the fluence and absorbed dose along the central axis are closely correlated with that of the fluence and absorbed dose of the broad beam. The macroscopic cross section remains almost constant, quasi-independent of the energy up to the practical range of the primary particle. The enhanced lateral spread, caused by the decrease in energy, will reduce the fluence and absorbed dose along the central axis of the projectile. For example, only $20 \%$ of the primary lithium particles reach the Bragg peak at about $26 \mathrm{~cm}$ in water. The spatial fluence and LET distribution of these few lithium particles are quite homogeneously distributed within radii of approximately $2 \mathrm{~mm}$. The spatial distribution of the heavier fragments is, similar to that of the primary particles, significantly influenced by increasing energy losses at the end of the particle range. These few particles are of a high LET character, which means that severe DNA damage starts to appear which the cell may not be able to repair, causing increased cell damage and eventual cell kill. In treatment planning, these processes have to be taken into account. The analytical mean LET values of the primary ${ }^{12} \mathrm{C}$ in a $279 \mathrm{MeV} / \mathrm{u}$ carbon beam, derived from the primary mean energy depth dependency [17], is in agreement with previous values found both experimentally and by MC studies [36,37]. Also the analytically derived spatial distribution of the primary ${ }^{12} \mathrm{C}$ and its associated boron fragments in high energy 290 and $400 \mathrm{MeV} / \mathrm{u}$ carbon beams are in good agreement with recent semianalytical and experimental data [34,37].

The close agreement between the present analytical results for the primary particles and their associated fragments obtained with $\mathrm{MC}$ and experimental data indicates the accuracy of using the new solution of the Boltzmann equation for the primary particles and the transport of the generated fragments. This also illustrates the applicability of the recently developed mean energy formula for stopping power and mass-scattering power calculations and the new energy-range relations for primary and generated fragments $[16,17]$. Because of the small variation in energy loss of the primary ions, the range straggling term was disregarded for simplicity. This approximation holds well for high charge and mass of the ion. In order to take the influence of range straggling on the local mean energy spread in high-energy beams into account, the analytical transport can most simply be described by taking the standard deviation of the particle range into account, cf. [17]. The general small lateral spread of these light ions makes pencil beam kernels much more insensitive to tissue heterogeneities whereas the ranges are significantly influenced $[38,39]$. In order to predict the dose distribution in inhomogeneous media, such as the anatomy of the patient, the model for range scaling in tissuelike media may then be useful [17] in combination with present fluence and absorbed dose expressions. To summarize, the present analytical approaches are consistent with MC and experimental data and could be very useful for fast calculation of the dose from the primary and associated fragments generated in therapeutic light ion pencil beams. The analytical theories could also be useful for treatment verification by means of in vivo PET imaging.

\section{ACKNOWLEDGMENTS}

Valuable suggestions and discussions with Jiping Xin, Professor Mohammad Asadzadeh, and Dževad Belkić are gratefully acknowledged. Also acknowledged is Martha Hultqvist for providing statistical information of Monte Carlo.

[1] H. Tsujii et al., New J. Phys. 10, 075009 (2008).

[2] J. Kempe, I. Gudowska, and A. Brahme, Med. Phys. 34, 183 (2007). 
[3] E. Fermi, Rev. Mod. Phys. 13, 265 (1941).

[4] L. Eyges, Phys. Rev. 74, 1534 (1948).

[5] A. Brahme, National Institute of Radiation Protection, Stockholm, Sweden, Report SSI (1975-011) (1975).

[6] A. Brahme, I. Lax, and P. Andreo, Acta Radiol. Oncol. 20, 147 (1981).

[7] K. R. Hogstrom, M.D Mills, and P. R. Almond, Phys. Med. Biol. 26, 445 (1981).

[8] ICRU Report No. 35, Bethesda, MD (1984).

[9] L. Zheng-Ming and A. Brahme, Radiat. Phys. Chem. 41, 673 (1993).

[10] A. Carlsson, P. Andreo, and A. Brahme, Phys. Med. Biol. 42, 1033 (1997).

[11] K. R. Russel et al., Phys. Med. Biol. 45, 9 (2000).

[12] M. Hollmark, J. Uhrdin, Dž. Belkić, I. Gudowska, and A. Brahme, Phys. Med. Biol. 49, 3247 (2004).

[13] N. Kanematsu, T. Akagi, Y. Takatani, S. Yonai, H. Sakamoto, and H. Yamashita, Phys. Med. Biol. 51, 4807 (2006).

[14] C. J. Mertens, J. W. Wilson, A. A. Walker, and J. Tweed, Adv. Space Res. 40, 1357 (2007).

[15] M. Hollmark, I. Gudowska, Dž. Belkić, A. Brahme, and N. Sobolevsky, Phys. Med. Biol. 53, 3477 (2008).

[16] J. Kempe and A. Brahme, Phys. Med. 26, 6 (2010).

[17] J. Kempe and A. Brahme, Med. Phys. 35, 159 (2008).

[18] I. Gudowska, N. Sobolevsky, P. Andreo, Dz. Belkić, and A. Brahme, Phys. Med. Biol. 49, 1933 (2004).

[19] O. Geithner, P. Andreo, N. Sobolevsky, G. Hartmann, and O. Jäkel, Phys. Med. Biol. 51, 2279 (2006).

[20] A. Brahme, in Computed Electron Beam Dose Planning, edited by A. Brahme (Acta. Radio, Stockholm, 1983), Suppl. 364, pp. 11-19.

[21] L. Sihver, C. H. Tsao, R. Silverberg, T. Kanai, and A. F. Barghouty, Phys. Rev. C 47, 1225 (1993).
[22] D. Schardt et al., Adv. Space Res. 17, 87 (1996).

[23] A. Fukumura et al., in Advances in Hadrontherapy (Elsevier, Amsterdam, 1997), pp. 325-330.

[24] H. Bichsel, T. Hiraoka, and K. Omata, Radiat. Res. 153, 208 (2000).

[25] A. S. Goldhaber, Phys. Lett. 53B, 306 (1974).

[26] D. E. Greiner, P. J. Lindström, H. H. Heckman, B. Cork, and F. S. Beiser, Phys. Rev. Lett. 35, 152 (1975).

[27] N. Matsufudji et al., Phys. Med. Biol. 50, 3393 (2005).

[28] J. M. Kidd, P. J. Linstrom, H. J. Crawford, and G. Woods, Phys. Rev. C 37, 2613 (1988).

[29] M. Giacomelli, L. Sihver, J. Skvarc, N. Yasuda, and R. Ilić, Phys. Rev. C 69, 064601 (2004).

[30] M. Giacomelli, Ph.D. thesis, University of Ljubljana, Slovenia, 2004.

[31] A. S. Botvina, A. V. Dementyev, O. N. Smirnova, N. M. Sobolevsky, and V. D. Toneev, in International Codes and Model Intercomparison for Intermediate Energy Activation Yields, edited by R. Michel and P. Nagel [NSC/DOC (97)-1, NEA/P\&T No. 14, OECD, Paris, 1997], pp. 307-312.

[32] ICRU Report No. 49, Bethesda, MD (1993).

[33] ICRU Report No. 73, Oxford University Press (2005).

[34] E. Haettner, M.Sc. thesis, Royal Institute of Tecnology, Stockholm, Sweden, 2006.

[35] N. Matsufuji, A. Fukumura, M. Komori, T. Kanai, and T. Kohno, Phys. Med. Biol. 48, 1605 (2003).

[36] I. Gudowska, J. Kempe, and N. Sobolevsky, Radiat. Prot. Dos. 122, 483 (2006).

[37] T. Inaiwiva, T. Furukawa, N. Matsufuji, T. Kohno, S. Sato, K. Noda, and T. Kanai, Phys. Med. Biol. 52, 7261 (2007).

[38] M. Krämer, O. Jäkel, T. Haberer, G. Kraft, D. Schardt, and U. Weber, Phys. Med. Biol. 45, 3299 (2000).

[39] O. Jäkel, Phys. Med. Biol. 51, N173 (2006). 